\title{
Surface waves simulation in porous media by domain decomposition
}

\author{
Yu Zhang, Shuangxi Zhang \\ School of Geodesy and Geomatics, \\ Key Laboratory of Geospace Environment and Geodesy \\ Ministry of Education, \\ Collaborative Innovation Center for Geospatial Technology, \\ Wuhan University
}

\author{
Wuhan, China \\ yuzhang@sgg.whu.edu.cn; yuz124@gmail.com \\ Ping Ping \\ State Key Laboratory of Geodesy and Earth's Dynamics \\ Institute of Geodesy and Geophysics, CAS \\ Wuhan, China
}

\begin{abstract}
A numerical approach for free surface boundary formulation is proposed for simulation of surface waves in porous media, based on the Biot poroelastic model. A set of free surface characteristic variables for incoming and outgoing wave fields are set by domain decomposition for solid and fluid velocity and stress equations to account for the discontinuity of variables along the interface. A numerical example of a pseudospectral approach using Fourier and Chebyshev methods to compute the spatial derivatives along the horizontal and vertical directions, respectively and a splitting algorithm time solver accounting for the stiffness of the differential equations is presented to show the computional efficiency of the proposed method.
\end{abstract}

Keywords—surface wave; poroelastic; domain decomposition; numerical simulation

\section{INTRODUCTION}

Because of the presence of fluids in near surface earth media, a single phase pure elastic model is insufficient in describing the corresponding seismic wave propagation phenomena. Wave motion in a more complex composite medium containing fluids of gas and/or liquid filling the pore space can be described in poroelastic model based on Biot's theory $^{[1]}$. In the theory, the characteristics of wave propagation can be used to infer fluid properties such as water distribution and permeability that are useful in many science and engineering fields. Similarly, numerical simulation of wave propagation in poroelastic media is therefore crucial and basic for near-surface studies using seismic waves. Many works on this subject including finite difference ${ }^{[2]}$, finite element ${ }^{[3]}$ and spectral element ${ }^{[4]}$ approaches are progressed.

Due to discontinuity of variables along the free surface interface, simulation of surface wave is more complex than body wave, especially for the spatial operator perpendicular to the interface. Previously, many methods are proposed for elastic media. For instance, the direct and simple vacuum formulism in which the boundary conditions are assumed to be implicitly fulfilled by the discontinuity of medium parameters on the nodes. For finite difference method, another popular method is the imaging method, where the stress variables are imaged as odd functions across an interface. Several explicit medium averaging approaches are also proposed for finite difference method, where a fictitious layer is always presented to simulate the traction-free effects on surface waves. For spectral element and finite element method, the free surface

This research is kindly supported by "973-project” (No. 2013CB733303), NSFC (No. 41304077), Postdoctoral Science Foundation of China (No.2014T70740). boundary is automated satisfied in integration formulation, however the complex effects of fluids through the free surface ${ }^{[5-6]}$ make the modeling of surface waves along free surface interface of a poroelastic medium is more challenging than that for elastic media.

In this study, we present a numerical algorithm that allows for the accurate simulation of surface wave propagation in porous media. The algorithm is based on an explicit treatment of interfaces. A decomposed domain is used for the wavefield at the interface to describe an incoming and an outgoing field for the medium based on characteristic variables that are evaluated for free surface boundary conditions ${ }^{[7]}$ and has the advantage that it accounts for variables that are discontinuous across interfaces and hence cannot be correctly approximated with a gradient ${ }^{[4]}$. The Chebyshev operator is used in the vertical direction for a denser sampling in the vicinity of the surface, which is necessary for accurate modeling of surface wave propagations ${ }^{[8]}$. The stiff part in the Biot poroelastic differential equations is splitted to solved analytically due to presence of a diffusive slow mode at low frequencies, and the regular part is solved by an explicit fourth-order Runge-Kutta algorithm in a splitting time integration method ${ }^{[9]}$.

\section{EQUATIONS FOR POROELASTICITY}

\section{A. Constitutive Relations}

Based on Biot's poroelastic model ${ }^{[1]}$, the stress and strain relation in a two dimensional plane strain problem can be written as

$$
\begin{gathered}
\tau_{i j, t}=\mu\left(v_{i, j}+v_{j, i}\right)+\delta_{i j}\left(\lambda_{u} v_{i, i}+\alpha M q_{i, i}\right), \\
p_{, t}=-\alpha M v_{i, i}-M q_{i, i},
\end{gathered}
$$

where $\tau_{i j}$ is the solid stress, $p$ is the pore fluid pressure; $v_{i}$ and $q_{i}$ are the solid particle velocity and fluid particle velocity relative to the frame, respectively; $\lambda_{u}$ is the undrained first Lamé coefficient which equals to $K_{m}+2 / 3 \mu+\alpha^{2} M ; \mu$ is the shear modulus. $\alpha$ and $M$ are the Biot coefficients, which are defined as

$$
\alpha=1-\frac{K_{m}}{K_{s}}
$$




$$
M^{-1}=\frac{\alpha-\phi}{K_{s}}+\frac{\phi}{K_{f}},
$$

\section{B. Biot-Euler Equations}

$$
\tau_{i j, j}=\rho v_{i, t}+\rho_{f} q_{i, t},
$$

where $\rho=\rho_{s}(1-\phi)+\rho_{f} \phi, \rho_{s}$ and $\rho_{f}$ are the densities of solid grains and pore fluid.

\section{Darcy-Euler Equations}

$$
p_{, i}=\rho_{f} v_{i, t}+m q_{i, t}+b q_{i},
$$

where $m=C \rho_{f} / \phi, C$ is the tortuosity, and $b$ is the Darcy coefficient, which can be written as $b=\eta / \kappa$ at seismic frequencies, where $\eta$ denotes the viscosity of the pore fluid and $\kappa$ is the dynamic permeability of the porous medium.

\section{CHARACTERISTIC VARIABLES IN DECOMPOSED DOMAINS FOR BOUNDARY CONDITIONS}

The boundary conditions are implemented by using a explicit characteristic variables in decomposed domains ${ }^{[7] 10]}$. This method has modeled free surface, internal interface and no-reflecting boundary conditions. The wave equations are written in decomposed domains into incoming and outgoing wave modes. For satisfying the boundary conditions of the variables, the incoming waves are constrained by the boundary conditions and the outgoing waves are determined by the full space solutions, whose idea is similar to the imaging approach in finite difference method.

The characteristic variables for the solid and fluid in the porous medium can be written in characteristic vector. If we assume the interface is perpendicular to $\mathrm{z}$ axis in two dimension, which is also valid for the inclined cases, the regular propagation part of poroelastic differential equation can be rewritten by

$$
\mathbf{v}_{, t}=\mathbf{A} \mathbf{v}_{, x}+\mathbf{B} \mathbf{v}_{, z}+\mathbf{s}
$$

where $\mathbf{v}=\left[v_{x}, v_{z}, q_{x}, q_{z}, \tau_{x x}, \tau_{z z}, \tau_{x z},-p\right]^{T}, \mathbf{A}$ and $\mathbf{B}$ are propagation matrixes in porous medium. $\mathbf{s}$ is the source term in the equation. And

$$
\begin{aligned}
& \mathbf{A}=\left(\begin{array}{cccccccc}
0 & 0 & 0 & 0 & a & 0 & 0 & b \\
0 & 0 & 0 & 0 & 0 & 0 & a & 0 \\
0 & 0 & 0 & 0 & b & 0 & 0 & c \\
0 & 0 & 0 & 0 & 0 & 0 & b & 0 \\
E_{u} & 0 & \alpha M & 0 & 0 & 0 & 0 & 0 \\
\lambda_{u} & 0 & \alpha M & 0 & 0 & 0 & 0 & 0 \\
0 & \mu & 0 & 0 & 0 & 0 & 0 & 0 \\
\alpha M & 0 & M & 0 & 0 & 0 & 0 & 0
\end{array}\right),
\end{aligned}
$$

$\mathbf{B}=$

$$
\left(\begin{array}{cccccccc}
0 & 0 & 0 & 0 & 0 & 0 & a & 0 \\
0 & 0 & 0 & 0 & 0 & a & 0 & b \\
0 & 0 & 0 & 0 & 0 & 0 & b & 0 \\
0 & 0 & 0 & 0 & 0 & b & 0 & c \\
0 & \lambda_{u} & 0 & \alpha M & 0 & 0 & 0 & 0 \\
0 & E_{u} & 0 & \alpha M & 0 & 0 & 0 & 0 \\
\mu & 0 & 0 & 0 & 0 & 0 & 0 & 0 \\
0 & \alpha M & 0 & M & 0 & 0 & 0 & 0
\end{array}\right),
$$

$E_{u}$ is the undrained $\mathrm{P}$ wave modulus, and

$$
a=\frac{m}{m \rho-\rho_{f}^{2}}, b=\frac{\rho_{f}}{m \rho-\rho_{f}^{2}}, c=\frac{\rho}{m \rho-\rho_{f}^{2}},
$$

interface
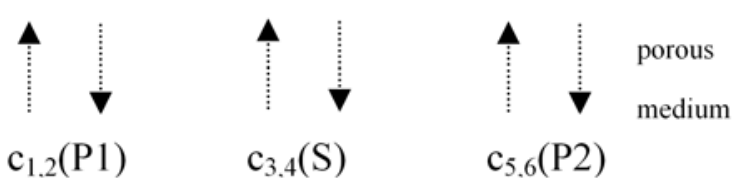

Fig. 1. The six characteristic variables for the three wave modes in the porous medium. The incoming variables $\left(\mathrm{c}_{2,4,6}\right)$ are calculated in the decomposed domain by boundary conditions.

The characteristic variable vector is given by ${ }^{[10]}$,

$$
\mathbf{c}=\mathbf{L v},
$$

where $\mathbf{L}$ is the matrix each row of which is eigenvector of the matrix $\mathbf{B}$. Vector $\mathbf{c}$ can be drawn from wave equation as

$$
\mathbf{c}_{, t}=\Lambda \mathbf{c}_{, z},
$$

where the diagonal matrix $\boldsymbol{\Lambda}$ is written by

$$
\Lambda=\mathbf{L B L}^{-1},
$$

The non-zero eigenvalues corresponds to three incoming and outgoing wave modes in the porous medium (Fig.1). The characteristic variable vector c can hence be obtained by the conbinations of eigenvectors in $\mathrm{L}$ and propagation variables in $\mathrm{V}$.

By decomposing c into incoming and outgoing wave fields on the defined interface grid contacts, the variables of c, a part calculated from regular part of wave equation (7), and a part calculated from whatever possible boundary conditions for porous medium ${ }^{[5]}$, then present the wave propagation behaviors on the interface, which is desirable for surface wave phenomena. This approach can be applied to represent the any variable discontinuity across the interface for various grid definations $^{[4]}$.

\section{Modeling ALgORITHM}

We use pseudospectral method as an example, which is an efficient and accurate wave modeling technique ${ }^{[3]}$. In this approach, the spatial differential operators are implemented by 
spectral technique incorporation of boundary condition set for characteristic variables and time integration. In the horizontal direction, the Fourier pseudospectral method is adopted since it is simple and efficient in terms of uniform interval of grids per wavelength. However, the Fourier method is not the only option for the domain decomposition with source and receivers closed to the free surface, for free surface can only be modeled by vacuum 'zero-padding' approach above the interface to circumvent the spurious reflection boundary, which is not optimal for surface wave modeling. An alternative method is to use the Chebyshev method to compute vertical directional spatial derivative in the wave equations ${ }^{[7]}$. The Chebyshev method is not periodic as the Fourier method and allows for the incorporation of the characteristic variable decomposition discussed in the last section, including freesurface, internal interface and non-reflecting conditions at the bottom of the mesh.

To improve the conventional Chebyshev method with two major disadvantages, we use a mapping transform for the vertical coordinate to stretch the mesh and increase the minimum grid interval, hence also increase the time step of the Runge-Kutta time integration algorithm, thus improving the time stable criterion from $O(\mathrm{~N} 2)$ to $O(\mathrm{~N})$ and breaking the limitation of Gauss-Lobatto interpolation points at interface definition $^{[7]}$. Furthermore, this transformation can be also used for spatial grid adaptation in the sense that the interpolation points can be redistributed and properly selected in regions with sharp velocity gradients, fine layering or complex interface geometries.

The slow wave mode that shows diffusive property in the Biot poroelasticity makes the differential equation stiff, as the whole system possesses two severely different time scale behaviors. Therefore, a numerical solution of the Biot wave equation poses a serious stable problem. To overcome this problem, the differential equations are solved with the splitting algorithm introduced by Carcione \& Quiroga-Goode ${ }^{[11]}$. The numerical solution of the regular, non-stiff part of the differential equations is obtained by using a normal fourthorder Runge-Kutta method as a time-stepping algorithm. Meanwhile, the stiff part that corresponds to the diffusion phenomenon can be solved analytically as ${ }^{[11]}$

$$
\begin{gathered}
v_{i}^{*}=v_{i}^{n}-\rho_{f} \rho^{-1}\left[\exp \left(\lambda_{s} d t\right)-1\right] q_{i}^{n}, \\
q_{i}^{*}=\exp \left(\lambda_{s} d t\right) q_{i}^{n},
\end{gathered}
$$

where $\lambda_{s}=(\eta / \kappa) c$, which is the slow wave eigenvalue in a viscous fluid system. These velocities are the input of the explicit time integration for the regular part.

\section{Simulation EXAMPLE}

We simulate the surface wave in a homogeneous halfspace by domain decomposition (DD) for the fully drained open pore condition ${ }^{[5]}$. The properties corresponds to a water saturated lightly consolidated sandstone are shown in TABLE I. The $30 \mathrm{~Hz}$ first derivate of a Gaussian wavelet with $0.05 \mathrm{~s}$ time shift is applied to generate the vertical stress disturbance in the poroelastic medium to simulate a hammer blow on the free surface. We tested the simulation of the proposed method compared with the generalized averaging (GA) approach ${ }^{[12]}$, using a forth order grid dispersion related finite difference operator $^{[13]}$.

Fig.2 shows the seismograms at offset $80 \mathrm{~m}$ of the vertical component of solid particle velocities and pore fluid pressure for the body waves (left panels) and surface waves (right panels) by both the DD and GA approaches. The results show that both approaches have equivalent precision for both body and surface waves. Pseudospectral simulation may have higher precision. Fig. 3 shows four snapshots at $0.12 \mathrm{~s}$, of the vertical component of solid particle velocities and pore fluid pressure for the DD approaches. Fast P - P1 wave, S wave and P2 wave can be identified in the internal, and one type of Rayleigh - R1 wave can be identified near surface. Only compressional components can be observed in the panels of pore fluid pressure.

\begin{tabular}{|c|c|c|}
\hline Parameter & & \\
\hline Pore fluids & & Water \\
\hline Bulk modulus of fluid (GPa) & $K_{f}$ & 2.25 \\
\hline Density of fluid $\left(\mathrm{kg} / \mathrm{m}^{3}\right)$ & $\rho_{f}$ & 1000 \\
\hline Viscosity $\left(10^{-3} \mathrm{Ns} / \mathrm{m}^{2}\right)$ & $\eta$ & 0 \\
\hline Porous media & & Lightly consolidated sand \\
\hline Bulk modulus of frame (GPa) & $K_{m}$ & 0.621 \\
\hline $\begin{array}{c}\text { Shear modulus of frame } \\
\text { (GPa) }\end{array}$ & $\mu$ & 0.455 \\
\hline Bulk modulus of solid (GPa) & $K_{s}$ & 36 \\
\hline Density of solid $\left(\mathrm{kg} / \mathrm{m}^{3}\right)$ & $\rho_{\mathrm{s}}$ & 2650 \\
\hline Porosity & $\phi$ & 0.3 \\
\hline $\begin{array}{l}\text { Intrinsic permeability }\left(10^{-}\right. \\
\left.{ }^{13} \mathrm{~m}^{2}\right)\end{array}$ & $\kappa$ & 10 \\
\hline
\end{tabular}

TABLE I. Physical Properties For a Poroelastic Medium

\section{DISCUSSION AND CONCLUSIONS}

We apply domain decomposition to handle the boundary condition in order to simulate the surface wave propagation in a porous medium. Numerical tests indicate that the surface wave can be simulated properly compared with staggered finite difference generalized averaging approach. This method is physically straightforward, and can be applied in various numerical methods as finite element, spectral element and several central finite difference (e.g. ADER) methods to represent any interface effect in the medium of wave propagation phenomenon. 

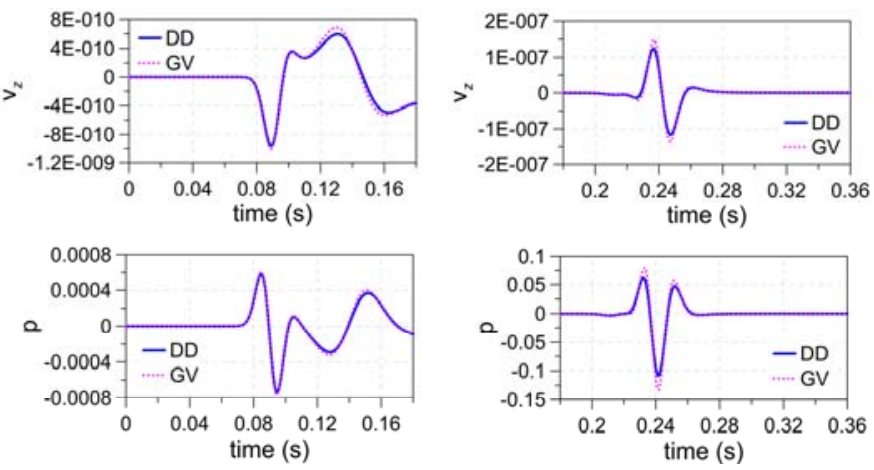

Fig. 2. Seismograms of vertical component of solid particle velocities (upper panels) and pore fluid pressure (lower panels) for body waves (left panels) and the surface waves (right panels).
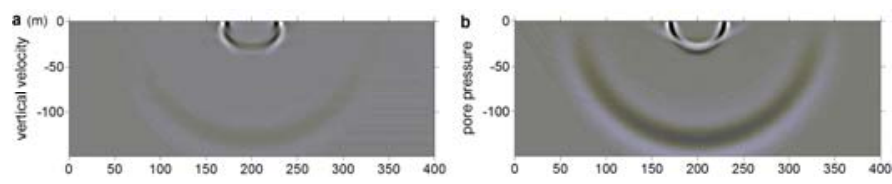

Fig. 3. Snapshots at $0.12 \mathrm{~s}$ of the vertical component of solid particle velocities (a) and pore fluid pressure (b) obtained by DD.

\section{REFERENCES}

[1] M.A. Biot, Mechanics of deformation and acoustic propagation in porous media: J. App. Physics, 1962, 33, 1482-1498.
[2] Y. J. Masson, S. R. Pride and K. T. Nihei, Finite difference modeling of Biot's poroelastic equations at seismic frequencies: J. Geophy. Res., 2006, 111, B10305.

[3] S. Picotti, J. M. Carcione, J. G. Rubino, and J. E. Santos, P-wave seismic attenuation by slow-wave diffusion: Numerical experiments in partially saturated rocks: Geophysics, 2007, 72, N11-N21.

[4] C. Morency and J. Tromp, Spectral-element simulations of wave propagation in porous media: Geophys. J. Int., 2008, 175, 301-345.

[5] Y. Zhang, Y. Xu and J. Xia, Analysis of dispersion and attenuation of surface waves in poroelastic media in the exploration seismic frequency band: Geophys. J. Int., 2011, 182, 870-888.

[6] Y. Zhang, Y. Xu and J. Xia, Wave fields and spectra of Rayleigh waves in poroelastic media in the exploration seismic frequency band: Adv. Water Res., 2012, 49, 62-71.

[7] J. M. Carcione, Modelinh anelastic singular surface waves in the earth, Geophysics, 1992, 57, 781-792.

[8] R. Mittet, Free-surface boundary conditions for elastic staggered-grid modeling schemes, Geophysics, 2002, 67, 1616-1623.

[9] J. Carcione, Wave Fields in Real Media: Wave Propagation in Anisotropic, Anelastic, Porous, and Electromagnetic Media, 2nd edn, Elsiever, 2007, Amsterdam.

[10] R. Sidler, J. M. Carcione and K. Holliger, Simulation of surface waves in porous media, Geophys. J. Int., 2010, 183, 820-832.

[11] J. M. Carcione and G. Quiroga-Goode, Some aspects of the physics and numerical modelling of Biot compressional waves, Journal of Computational Acoustics, 1995, 3(4), 261-280.

[12] Y. Liu and M. K. Sen, Scalar wave equation modeling with time- space domain dispersion-relation-based staggered-grid finite-difference schemes, BSSA, 2011, 101, 141-159.

[13] Y. Zhang, P. Ping, P. Deng, H. Zhou and S. Zhang, A free surface formulation for finite difference modeling of surface wave in porous media, 85th Annual International Meeting, SEG expanded abstract, 2406-2411, 2015. 